\title{
Eine vereinfachte Methode zur Bestimmung von Adrenalin und Noradrenalin im Harn
}

\author{
Von \\ KonRad LaUber \\ Aus dem Medizinisch-chemischen Institut der Universität Bern (Direktor: Prof. Dr. med. H. Aebi)
}

(Der Schriftleitung zugegangen am 8. November 1963)

\begin{abstract}
Eine Methode zur Isolierung von Noradrenalin und Adrenalin und deren fluorimetrische Bestimmung wird in allen Einzelheiten beschrieben. Das Verfahren benutzt die Erfahrungen von Euters sund NadeAus mit einigen Modifikationen und technischen Verbesserungen. Ein ,innerer" Katecholaminstandard wird verwendet. Die Vorund Nachteile der $2 \mathrm{pH}$-Methode und der 2 Filter-Methode (Fluoreszenzmessung an einer Probe bei zwei verschiedenen Wellenlängen des Primärlichts) werden einander gegenübergestellt. An Hand von 10 gesunden Versuchspersonen wird ein vorläufiger Normalbereich für die Adrenalịn- und Noradrenalinausscheidung aufgestellt.

The isolation and fluorimetric determination of noradrenalin and adrenalin is described in detail. The method is modified from those of von EULER and NADEAU with technical improvements. An "internal" catecholamine standard is used. The advantages and disadvantages of the $2 \mathrm{pH}$ method and the 2 filter method (fluorescence measurement on one sample at two different primary wavelengths) are compared. The range of adrenalin and noradrenalin excretion was determined for 10 healthy persons.
\end{abstract}

Die Bestimmung der Katecholamine gehört zu den anspruchsvollsten Analysen im klinisch-chemischen Labor; sie wird daher nur von wenigen Institutionen routinemäßig durchgeführt. In der vorliegenden Arbeit wird eine Methode beschrieben, die es auch dem Nichtspezialisten ermöglichen soll, die Urin-Katecholamine zu bestimmen. Das Verfahren lehnt sich an die von Euler und Floding (1) bzw. Nadeau und Sobolewsiki (2) beschriebenen Prinzipien an: Saure Hydrolyse der Konjugate; Adsorption an Aluminiumoxyd bei schwach alkalischem pH; Elution mit Säure; Úberführung der Amine in Lutine; Bestimmung der Lutinkonzentration mittels Fluorimetrie; Differenzierung von Adrenalin und Noradrenalin durch Oxydation bei verschiedenen $\mathrm{pH}$-Werten.

\section{Methodik}

Geräte

pH-Meter; Magnetrührer; Filternutsche; Zentrifuge; Photometer mit Zusatz zur Fluoreszenzmessung, z. B. „Eppendorf““ Stoppuhr.

Reagènzien

1. Schwefelsäure $10 n: 275 \mathrm{~m} l$ konz. $\mathrm{H}_{2} \mathrm{SO}_{4}$ mit $\mathrm{H}_{2} \mathrm{O}$ ad $1 l$.

2. Aluminiumoxyd „aktiv“, „sauer“ (Merck).

3. Natronlauge/Komplexon: $30 \mathrm{~g}$ Natriumhydroxyd $+2 \mathrm{~g}$ Komplexon III (Dinatriumsalz der Äthylendiamin-Tetraessigsäure) in Wasser auflösen und auf $2 \dot{l}$ auffüllen. (In Glasgefäß aufbewahren.)

4. Zitronensäure/Zinksulfat: $35 \mathrm{~g}$ Zitronensäure $\left(\mathrm{C}_{6} \mathrm{H}_{8} \mathrm{O}_{7}\right.$. $\left.\mathrm{H}_{2} \mathrm{O}\right)+0,5 \mathrm{~g}$ Zinksulfat $\left(\mathrm{ZnSO}_{4} \cdot 7 \mathrm{H}_{2} \mathrm{O}\right)$ in Wasser lösen und auf $1 l$ auffüllen.

5. Natriumbicarbonat 6,6 proz.

6. Kaliumferricyanid 0,2 proz.
7. Natronlauge 10 proz. (in Glasflasche aufbewahren).

8. Ascorbinsäure 1 proz. (jeden Tag frisch ansetzen).

9. Ascorbat/Natronlauge: Unmittelbar vor Gebrauch $0,4 \mathrm{~m} l$ von Lösung 8 und $24 \mathrm{ml}$ von Lösung 7 mischen.

10. Fluoteszenzstandard: Chininsulfatlösungen von 2,5-, 5-, 10-, 25 - und $50 \mathrm{mg} \%$ in $0,1 n$ Schwefelsäure.

11. Katecholaminstandard: $15 \mathrm{mg}$ Adrenalin-Base $+90 \mathrm{mg}$ Noradrenalin-Bitartrat-Monohydrat (z. B. von „Fluka“, Buchs, St. Gallen) in $5 \mathrm{~m} l 1 n \mathrm{HCl}$ auflösen und mit Wasser auf $500 \mathrm{~m} l$ verdünnen. (Gehalt $30 \mu \mathrm{g}$ Adrenalin bzw. $90 \mu \mathrm{g}$ Noradrenalin pro $\mathrm{m} l$ ). Lösung in kleinen Portionen einfrieren; jeden Tag eine neue Portion auftauen.

- Etwa einmal pro Monat sollte kontrolliett werden, ob die Standardlösung noch die ursprüngliche Fluoreszenz ergibt: $\mathrm{Ca} 10 \mathrm{ml}$ Zitronensäure 4 werden mit Bicarbonat $5 \mathrm{am} \mathrm{pH}-$ Meter auf pH 6 neutralisiert. Je $20 \mu l$ Standardlösung werden mit $1,5 \mathrm{~m} l$ dieses Puffers resp. $1,5 \mathrm{~m} l$ unveränderter Zitronensäure 4 gemischt. Dann wird mit Ferricyanid und Ascorbat entwickelt wie unten beschrieben (vgl. Schema). Die Fluoreszenz der beiden Proben wird gegen Chininsulfat von $25 \mathrm{mg} \%$ abgelesen. Sind die Flluoreszenzwerte gegenüber dem frisch hergestellten Standard um mehr als $5 \%$ gesunken, dann ist neue Lösung herzustellen.

\section{Arbeitsweise}

\section{Harnsammeln}

Ca $5 \mathrm{ml}$ konz. Schwefelsäure oder 70proz. Perchlorsäure werden ins leere Sammelgefäß gegeben. Der Urin muß zur Verhinderung von Autoxydation von Anfang an sauer sein. Die Tagesmenge wird ermittelt und ein Aliquot davon verarbeitet.

\section{Hydrolyse}

In 6 Reagensgläschen werden je $12 \mathrm{~m} l$ Urin mit $1 \mathrm{ml}$ Schwefelsäure 1 gemischt. Zwei Gläschen werden außer- 
dem mit $20 \mu l$ und zwei mit $60 \mu l$ Standardlösung versetzt. Die Gläschen werden mit kleinen Bechern abgedeckt und 20 Min. ins kochende Wasserbad gestellt. Die Proben werden dann auf Leitungswassertemperatur abgekühlt.

\section{Adsorption|Elution}

(vorläufig werden nur die Proben ohne und jene mit $20 \mu l$ Standard zu Ende verarbeitet)

$5 \mathrm{ml}$ der gekochten Urinproben werden in $50 \mathrm{ml}$ Bechergläser übergeführt. Unter kräftigem Rühren (Magnetrührer) werden etwa $10 \mathrm{~m} l$ Natronlauge/Komplexon 3 zugetropft. Am pH-Meter wird dann mit der gleichen Lösung auf $\mathrm{pH} \sim 4$ weiter neutralisiert. $0,5 \mathrm{~g}$ Aluminiumoxyd 2 wird zugesetzt. Mit einigen Tropfen von Lösung 3 wird das $\mathrm{pH}$ vorsichtig auf 8,5 verschoben. Beim ersten Erreichen dieses Wertes wird die Stoppuhr gestartet. Während $4 \mathrm{Min}$. wird unter ständigem Rühren das $\mathrm{pH}$ auf 8,5 konstant gehalten. Eine Spontanverschiebung nach unten wird durch weiteren Laugenzusatz kompensiert. Steigt das $\mathrm{pH}$ irrtümlicherweise auf über 8,7, dann ist die Probe mit weiteren $5 \mathrm{ml}$ gekochtem Harn frisch anzusetzen. Nach Ablauf der 4 Min. wird der Becherinhalt auf eine Nutsche mit relativ hartem Papierfilter (z. B. „Green Hydro" 803; Bittmann, Basel) gegeben und mit Vakuum abgesaugt. Das Becherglas wird zweimal mit $10 \mathrm{~m} l$ Wasser nachgespült. Unmittelbar nach dem letzten Trockenlaufen des Filters wird ein Reagensgläschen unter die Nutsche gestellt und $1,5 \mathrm{~m} l$ Zitronensäure/ Zinksulfat 4 aufs Filter gegeben. Während 0,5 Min. wird die Nutsche bewegt, so daß das Aluminiumoxyd aufgewirbelt wird. Das Eluat wird abgesaugt und der Elutionsprozeß $\beta$ weimal mit $1,5 \mathrm{ml}$ Zitronensäure 4 wiederholt (je 0,5 Min. bewegen vor dem Absaugen).

\section{Lutinbildung}

Von jedem der vier zu einem Urin gehörenden Eluate werden $3 \mathrm{mal}$ je $1 \mathrm{ml}$ in 3 Zentrifugengläschen mit den Bezeichnungen 6, 3, 0 übergeführt. Mit den 12 Gläschen wird weiter verfahren wie in untenstehendem Schema angegeben.

Schema für die Lutinbildung

\begin{tabular}{l|c|c|c}
\hline & 6 & 3 & 0 \\
\hline Eluat & $1 \mathrm{~m} l$ & $1 \mathrm{~m} l$ & $1 \mathrm{~m} l$ \\
Bicarbonat 5 & $0,5 \mathrm{~m} l$ & $-\overline{\mathrm{m} l}$ & $-\overline{\mathrm{m} l}$ \\
Wasser & - & $0,5 \mathrm{~m} l$ & $0,1 \mathrm{~m} l$ \\
Kaliumferricyanid 6 & $0,1 \mathrm{~m} l$ & $0,1 \mathrm{~m} l$ & $0,1 \mathrm{~m}$ \\
\hline
\end{tabular}

schütteln und zwischen Ferricyanid- und Laugenzusatz genau 3 Min. stehenlassen

\begin{tabular}{l|c|c|c}
\hline Ascorbat/Natronlauge 9 & $3 \mathrm{~m} l$ & $3 \mathrm{~m} l$ & - \\
Natronlauge 7 & - & - & $3 \mathrm{~m} l$ \\
\hline
\end{tabular}

gut mischen, zentrifugieren

\section{Fluoreszenzmessung}

Die Messung wird hier für das „Eppendorf“-Photometer beschrieben. Folgende Filter werden verwendet: Primärfilter $405 / 436 \mathrm{~m} \mu$; Sekundärfilter $500-3000 \mathrm{~m} \mu$. Mit Chininsulfatlösung von $2,5 \mathrm{mg} \%$ wird die Fluoreszenz durch Regulierung der Photometerverstärkung auf 100 Skalenteile eingestellt. Gegen diesen Bezugswert werden alle 12 Proben abgelesen. Ist die Fluoreszenz der Proben „St-6" höher als 100, dann wird eine stärkere Chininsulfatlösung als Bezug verwendet.

\section{Schema für die Fluoreszenzmessung}

\begin{tabular}{c|c|c|c|c}
\hline & $\mathrm{St}_{1}$ & $\mathrm{St}_{2}$ & $\mathrm{~A}_{1}$ & $\mathrm{~A}_{2}$ \\
\hline 6 & & & & \\
\hline 3 & & & & \\
\hline 0 & & & & \\
\hline \hline $\mathrm{I}=6-3$ & & & & \\
\hline $\mathrm{II}=3-0$ & & & & \\
\hline
\end{tabular}

$A_{1}$ bzw. $A_{2}$ : Urin-Doppelproben ohne Standardzusatz; $\mathrm{St}_{1}$ bzw. $\mathrm{St}_{2}$ : Urin-Doppelproben mit Standardzusatz; 6: Bei $\mathrm{pH} 6$ oxydiert; 3: Bei $\mathrm{pH}<3$ oxydiert; 0: Blindfluoreszenz.

\section{Berechnung:}

(bei Verwendung der Proben mit $20 \mu l$ Standardzusatz)

$$
\begin{array}{cc}
\mu \mathrm{g} \% \text { Noradrenalin: } \quad \frac{\mathrm{I}_{\mathrm{A}}}{\mathrm{I}_{\mathrm{St}}-\mathrm{I}_{\mathrm{A}}} \cdot 15 \\
\mu \mathrm{g} \% \text { Adrenalin: } \frac{\mathrm{II}_{\mathrm{A}}-0,1 \cdot \mathrm{I}_{\mathrm{A}}}{\frac{\mathrm{II}_{\mathrm{St}}-0,1 \cdot \mathrm{I}_{\mathrm{St}}-\left(\mathrm{II}_{\mathrm{A}}-0,1 \cdot \mathrm{I}_{\mathrm{A}}\right)}{4}} \cdot 5 .
\end{array}
$$

\section{Diskussion}

1. Durch das Kochen des stark sauren Urins werden die konjugierten Amine gespalten und damit der Bestimmung zugänglich gemacht. Eine Bestimmung der gesamten Katecholamine erscheint uns zuverlässiger als die Analyse des freien Anteils, da durch das obligatorische Ansäuern des Urins während des Sammelns die Hydrolyse ohnehin schon ingangkommen kann (3).

2. Die Verwendung eines sog. inneren Standards ist unbedingt notwendig, weil die beim AdsorptionsElutionsprozeß entstehenden Verluste von Urin zu Urin stark schwanken. Auch der sog. Quenching-Effekt ist von Probe zu Probe verschieden. Der Zusatz von $20 \mu l$ Standardlösung zu $12 \mathrm{ml}$ Harn entspricht einer Konzentration von $5 \mu \mathrm{g} \%$ Adrenalin und $15 \mu \mathrm{g} \%$ Noradrenalin, auf den Urin bezogen. Bei einem normalen Harn erhält man dann für die Proben mit Standardzusatz etwa doppelt so hohe Fluoreszenzen wie für die Proben ohne Standard, was ein Optimum an Genauigkeit gewährleistet.

Wird der Quotient $\frac{I_{A}}{I_{S t}-I_{A}}$ größer als 3, was nur bei pathologischem oder hochkonzentriertem Urin vorkommt, dann ist der Analysengang mit den Proben, welche $60 \mu l$ Standard enthalten, zu wiederholen. Ist auch für den hohen Standardzusatz der relative Fluoreszenzunterschied zwischen "Standard" und „Analyse“ zu klein, dann wird der Urin vor der Hydrolyse zehnbis fünfzigfach. mit Wasser verdünnt. Das Endresultat ist sinngemäß zu vervielfachen. 
3. Die Abkühlung der gekochten Harnproben auf einige Grad unter Raumtemperatur empfiehlt sich, weil beim Neutralisieren auf $\mathrm{pH}=8,5$ so viel Wärme frei wird, $\mathrm{da} B$ die Zimmertemperatur wieder erreicht wird und sich eine Temperaturkorrektur am $\mathrm{pH}-$ Meter erübrigt.

4. Die Konzentration der Lauge wurde auf $1,5 \%$ angesetzt, weil dann das Endvolumen der auf $\mathrm{pH}=8,5$ eingestellten Probe die für den Adsorptionsprozeß optimale Größe erreicht (2). Das zugesetzte Komplexon verhindert die Bildung von ErdalkaliphosphatNiederschlägen, welche sich auf die Adsorption störend auswirken (2). Es empfiehlt sich, die Lauge über dem $\mathrm{pH}-\mathrm{Meter}$ aufzustellen und mittels Glasheber mit kurzem Polyvinylschlauch und Quetschhahn am unteren Ende in die Urinprobe tropfen zu lassen. Bei Verwendung von Polyäthylenflaschen für die $\mathrm{NaOH}$ wurden schlechtere Fluoreszenzausbeuten erhalten als bei $\mathrm{Ge}$ brauch von Glasflaschen (störender Weichmacher?).

5. Zur Filtration wurden früher von uns Glasfritten verwendet, welche sich aber schnell verstopften. Um für alle Proben die gleichen Bedingungen zu erhalten, mußten sie nach jeder Filtration umständlich gereinigt werden. Die von uns gegenwärtig verwendete Vakuumnutsche ist eine einfache Sonderanfertigung aus Polyäthylen, welche ein Festklemmen des Papierfilters gestattet (Abb. 1). Das Filtrieren und Nachwaschen

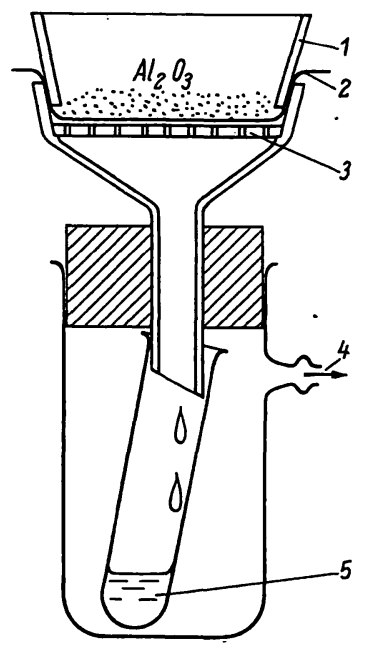

Abb. 1

Vakuumnutsche

1 = Satt sitzender Ring zum Festklemmen des Papierfilters

$2=$ Papierfilter 3 = Siebplatte $4=$ Vakuum $5=$ Eluat

hat möglichst rasch zu exfolgen, da die Katecholamine bei $\mathrm{pH}>6$ leicht autoxydabel sind. Für jede Filtration wird ein neues Filter verwendet. Die Filtration hat sich als unvergleichlich rascher .erwiesen als die Zentrifugation, wie sie von NADEAU (2) vorgeschlagen wird.

6. Zur Elution wird Zitronensäure den von andern Autoren $(2,4)$ vorgeschlagenen Säuren (Essigsäure, Schwefelsäure, Oxalsäure) vorgezogen, weil jene bei $\mathrm{pH}=6$ gut puffert und damit die Einstellung dieses
pH-Wertes wesentlich erleichtert. Das Zinksulfat, welches die Lutinbildung katalysiert, wird schon der Zitronensäure und nicht, wie in früheren Arbeiten beschreiben, vor dem Entwickeln jedem Gläschen separat zugesetzt.

7. Die Konzentration des Bicarbonats 5 ist so gewählt, da $\beta$ beim Mischen von $0,5 \mathrm{ml}$ mit $1 \mathrm{~m} l$ Eluat (nicht $1 \mathrm{~m} l$ Lösung 4!) $\mathrm{pH}=6,0$ resultiert. Bei jeder Neuherstellung von Lösung 4 bzw. 5 sollte mit dem pHMeter kontrolliert werden (Toleranz $\pm 0,2 \mathrm{pH}$-Einheiten).

8. Durch das Ferricyanid werden die Katecholamine in Adrenochrom bzw. Noradrenochrom übergeführt. Während die Bildung des Adrenochroms bei $\mathrm{pH}=6$ und in verdünnter Zitronensäure $(\mathrm{pH} \leqq 3)$ gleich verläuft, entsteht unterhalb $\mathrm{pH} 3$ nur etwa $8-10 \%$ Noradrenochrom $(100 \%$ bei $\mathrm{pH}=6)$. Durch Zusatz von Ascorbat in stark alkalischer Lösung werden Adrenochrom und Noradrenochrom in die fluoreszierenden Lutine übergeführt. Bei bloßem Laugenzusatz werden dagegen die oxydierten Katecholamine zerstört, und man erhält die Blindfluoreszenz der mitisolierten Begleitstoffe.

9. Für die Differenzierung von Adrenalin und Noradrenalin gibt es prinzipiell zwei Methoden: a) Oxydation der Katecholamine bei $\mathrm{pH}=6$ und $\mathrm{pH} \leqq 3$ und Fluoreszenzmessung mit ein und demselben Primärlicht (1); b) Oxydation nur bei pH 6 und Fluoreszenzmessung bei Anregung mit $z$ wei verschiedenen Wellenlängen $(2,5)$. Die beiden Methoden sind von uns verglichen worden. Die „Zwei-Filter-Methode" lieferte etwas weniger gut reproduzierbare Resultate als die „Zwei-pH-Methode", was sich schon rein theoretisch begründen läßt. Die Formeln lauten für die beiden Methoden:

Zwei $p H$ :

$$
\begin{aligned}
& \cdots \quad N=\frac{F_{6 A}-F_{3 A}}{F_{6 S t}-F_{3 S t}-\left(F_{6 A}-F_{3 A}\right)} \cdot K_{N} \\
& A=\quad \frac{F_{3 A}-F_{B}-0,1\left(F_{6 A}-F_{3 A}\right)}{F_{3 S t}-F_{B}-0,1\left(F_{6 S t}-F_{3 S t}\right)-\left[F_{3 A}-F_{B}-0,1\left(F_{6 A}-F_{3 A}\right)\right]} \cdot K_{A}
\end{aligned}
$$

Zwei Filter:

$$
\begin{aligned}
& N=\frac{q_{A}\left(F_{1 A}-F_{1 B}\right)-\left(F_{2 A}-F_{2 B}\right)}{q_{A}\left(F_{1 S t}-F_{1 A}\right)-\left(F_{2 S t}-F_{2 A}\right)} \cdot K_{N} \\
& A=\frac{q_{N}\left(F_{1 A}-F_{1 B}\right)-\left(F_{2 A}-F_{2 B}\right)}{q_{N}\left(F_{1 S t}-F_{1 A}\right)-\left(F_{2 S t}-F_{2 A}\right)} \cdot K_{A}
\end{aligned}
$$

$\mathrm{F}_{6}$ : Fluoreszenz bei $\mathrm{pH}=6$

$\mathrm{F}_{3}$ : Fluoreszenz bei $\mathrm{pH}=3$

$\mathrm{F}_{1}$ : Fluoreszenz bei Filter 1

$\mathrm{F}_{2}$ : Fluoreszenz bei Filter 2 (identisch mit $\mathrm{F}_{6}$ )

A: Urinprobe ohne Standardzusatz

St: Urinprobe mit Standardzusatz

B: Blindprobe mit zerstörtem Lutin

$\mathrm{K}_{\mathrm{N}}$ bzw. $\mathrm{K}_{\mathrm{A}}$ : Noradrenalin bzw. Adrenalinkonzentration des Standards

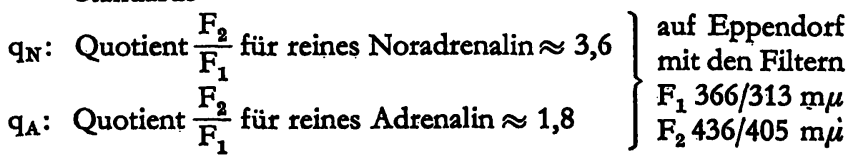


Bei beiden Methoden werden Fluoreszenzdifferenzen von zwei verschieden behandelten Proben $\left(F_{6 A}-F_{3 A}\right)$ bzw. $\left(F_{1 A}-F_{1 B}\right)$ usw. für die Berechnung verwendet. Es besteht also für beide Methoden eine ähnliche Gefahr der Summierung von 2 Ablesefehlern.

Annahme: Fluoreszenz von Noradrenalin und Adrenalin sowohl im Urin als auch im zugesetzten Standard gleich stark; zugesetzte Menge für beide Katecholamine gleich groß, wie die im Urin schon vorhandene Menge.

$$
\begin{gathered}
F_{6 A}-F_{3 A}=18 \text { (angenommen) } \\
\text { aus } F_{6 A}-F_{3 A} \text { berechnet: }
\end{gathered}
$$

\begin{tabular}{|c|c|}
\hline $\begin{array}{l}F_{6 S t}-F_{3} S t=36 \\
F_{3 A}-F_{B}=22 \\
F_{3 S t}-F_{B}=44\end{array}$ & $\begin{array}{l}\mathbf{F}_{1 \mathbf{A}}-\mathrm{F}_{1 \mathbf{B}}=16,7 \\
. . \\
\mathbf{F}_{1 \mathrm{St}}-\mathrm{F}_{\mathbf{1 A}}=16,7 \\
\mathbf{F}_{\mathbf{2 A}}-\mathrm{F}_{\mathbf{2 B}}=40 \\
\mathbf{F}_{\mathbf{2 S t}}-\mathbf{F}_{\mathbf{2 A}}=40\end{array}$ \\
\hline
\end{tabular}

Setzt man die Zahlen in obigen Formeln ein und rechnet mit einem Ablesefehler von +1 Skalenteil entweder bei $F_{3 A}$ oder $F_{6 A}$ bzw. entweder bei $F_{1 A}$ oder bei $F_{2 A}$, dann ergeben sich folgende $A b-$ weichungen vom richtigen Resultat:

$Z_{\text {wei }}$ pH: $\quad$ Noradrenalin; Fehler bei $\mathrm{F}_{6 \mathrm{~A}}$ : Abweichung $12 \%$ Fehler bei $\mathrm{F}_{3 \mathrm{AA}}$ : Abweichung 10,5\%

Adrenalin; Fehler bei $F_{3 A}$ : Abweichung 11,5\%

Zwet Filter: Noradrenalin; Fehler bei $\mathrm{F}_{2 \mathrm{~A}}$ : Abweichung 9,5\% Fehler bei $\mathrm{F}_{1 \mathrm{~A}}$ : Abweichung 42,5\%

Adrenalin; Fehlex bei $\mathrm{F}_{2 \mathrm{~A}}$ : Abweichung 22\% Fehler bei $F_{1 A}$ : Abweichung 31\%

Ablesefehler der $F_{1}$-Fluoreszenz wirken sich bei der „Zwei-FilterMethode" verheerend aus, wegen der Multiplikation durch die Faktoren $q_{N}$ bzw. $q_{A}$. Bei beliebigem Variieren der oben angenommenen Konzentrationsverhältnisse sind die durchschnittlichen Abweichungen bei gleichbleibenden Ablesefehlern bei der „ZweiFilter-Methode“ stets mindestens doppelt so groß wie bei der
„Zwei-pH-Methode“. Bei Verwendung der beiden Primärwellenlängen 335 und $385 \mathrm{~m} \mu$, wie von RITZEL (5) vorgeschlagen, liegen die Verhältnisse für die „Zwei-Filter-Methode“ auch nicht günstiger.

\section{Normalbereich}

Bei 10 gesunden Erwachsenen (5 Frauen und 5 Männer) wurde an je 3 fortlaufenden Tagen die Katecholaminausscheidung im Harn bestimmt. Als Mitteliverte der drei Bestimmungen wurden folgenden Zahlen erhalten:

\begin{tabular}{cccc}
\hline \multirow{2}{*}{ Frauen } & & $\begin{array}{c}\text { Noradrenalin } \\
\mu \mathrm{g} / 24 \text { Stdn. }\end{array}$ & $\begin{array}{c}\text { Adrenalin } \\
\mu \mathrm{g} / 24 \mathrm{Stdn} .\end{array}$ \\
& 1. & 172 & 16 \\
& 2. & 211 & 33 \\
& 3. & 133 & 36 \\
Männer & 4. & 155 & 5 \\
& 5. & 134 & 26 \\
& 6. & 159 & 34 \\
& 7. & 147 & 26 \\
& 8. & 210 & 15 \\
& 9. & 141 & 5 \\
& 10. & 198 & 23 \\
\hline
\end{tabular}

Mittel und Standardabweichung s: Noradrenalin $166 \pm 31 \mu \mathrm{g} /$ 24 Stdn.; Adrenalin $22 \pm 11 \mu \mathrm{g} / 24$ Stdn.

Normalbereich (Mittel $\pm 2 \mathrm{~s}$ ): Noradrenalin 104-228 $\mu \mathrm{g} / 24$ Stdn.; Adrenalin 0-44 $\mu \mathrm{g} / 24 \mathrm{Stdn}$.

Die Zahl der Versuchspersonen ist zu klein zur Aufstellung eines gültigen Normalbereiches. Eine Ausscheidung von weniger als $220 \mu \mathrm{g}$ Gesamt-Noradrenalin und weniger als $40 \mu \mathrm{g}$ Gesamt-Adrenalin dürfte aber als normal angesehen werden.

\section{Literatur}

1. von Euler, U. S. und I. Floding, Acta physiol. scand. 33, Suppl. 118, 45 (1955). - 2. NAdeAU, G. und G. Sobolewskr, J. Chromatogr. (Amsterdam) 6, 164 (1961). - 3. JACOBs, S. L., C. Sober und R. J. HeNRY, J. clin. Endocr., Springfield 21, 305
(1961). - 4. Pekrarinen, A. und M. E. Pitkänen, Scand. J. Clin. Laborat. Invest. 7, 1 (1955). - 5. Ritzer, G. und W. A. HuNzINGER, Klin. Wschr. 41, 419 (1963).
Dr. chem. Konrad Lauber Medizinisch-chemisches Institut der Universität Bern Bern/Schweiz, Bühlstr. 28 